\title{
Numerical simulation of a PCM shutter for buildings space heating during the winter
}

\author{
N. Soares ${ }^{1, *}$, A. Samagaio ${ }^{2}$, R.Vicente ${ }^{1}$, J. Costa $^{3}$ \\ ${ }^{1}$ Civil Engineering Department of the University of Aveiro, Aveiro, Portugal \\ ${ }^{2}$ Environment and Planning Department of the University of Aveiro, Aveiro, Portugal \\ ${ }^{3}$ ADAI - Mechanical Engineering Department of the University of Coimbra, Coimbra, Portugal \\ *Corresponding author. Tel: +351 234370845, Fax: +351 234370094,E-mail: nsoares@ua.pt
}

\begin{abstract}
The integration of Phase Change Materials (PCMs) into the building envelope provides a higher thermal inertia that, combined with the thermal insulation effect, can reduce the energy consumption.

Using a 2-dimensional simulation model based on the enthalpy formulation, a latent heat storage system has been numerically designed and parametrically optimized to take advantage of solar thermal energy for buildings space heating during the winter in Coimbra, Portugal. The main purpose of this study is to show the potential of incorporating PCMs in structural cells of shading elements associated to southward façade windows.

In view of the low thermal diffusivity of the Phase Change Material (PCM) chosen, the distance between metal fins is directly proportional to the energy storage/release capacity of the system. The results of the parametric study also show that solar radiation flux has a strong effect on the melting/charging process. On the other hand, the indoor temperature and the indoor heat convection transfer rate, during the night, play an important role in PCM solidification/discharging process.

In conclusion, an optimal thermal storage system - PCM shutter - can be designed for any given location and characteristic climatic data during the winter. The optimum depends strongly on the thermophysical properties of the PCM and on the internal boundary conditions considered.
\end{abstract}

Keywords: Phase Change Material, PCM, Enthalpy formulation, Energy storage, Numerical modelling.

\section{Nomenclature}

CM thickness of the metal fin

$E \quad$ total energy stored and released by the system during a day cycle.

$F \quad$ total PCM melted fraction..................... \%

FL thickness of the system ......................... $m$

$H \quad$ enthalpy of the system............................

$h \quad$ volumetric sensible enthalpy ............. $\mathrm{J} \mathrm{m}^{-3}$

$h_{e} \quad$ external convection heat transfer coefficient ............................ $\mathrm{W} \mathrm{m}^{-2}{ }^{\circ} \mathrm{C}^{-1}$

$h_{i} \quad$ internal convection heat transfer coefficient $W m^{-2}{ }^{o} C^{-1}$

$H L \quad$ inter-fins distance

\section{Introduction}

As referred by many authors [1-5], the thermal energy storage systems using PCMs have been recognized as one of the most advanced energy technologies to enhance the energy efficiency and sustainability of buildings. An interesting feature of PCMs is that they can store latent energy as well as sensible energy. Their high latent heat storage capacity combined with friendly energy systems employing endogenous energies, such as solar thermal energy, can reduce the energy consumption of buildings in a passive and sustainable way. The systems incorporating PCMs benefit also from the isothermal nature of the phase change process. For a review, see [1-5]. At the same time as the demand for thermal comfort in buildings in Portugal increases, so does the energy consumption for buildings' indoor environmental control, particularly, for space heating during the winter. The main goal of the present work is 
to design a latent thermal energy storage system incorporating PCM, taking advantage of solar energy, which is an abundant resource in Mediterranean climates, for space heating during the winter season in Coimbra, Portugal. The numerical simulation of the system is performed using a code specifically developed for this purpose, based on a 2-dimensional model following the enthalpy formulation.

The enthalpy formulation has been extensively employed by many authors in the modelling of phase change problems [6-11] and it is one of the most popular fixed-domain methods for solving the Stefan problem [4]. The problem of predicting the behaviour of phase change is difficult due to its nonlinear nature at the moving interface and, in addition, to the fact that the material at the two phases has different thermophysical properties. When the PCM undergoes a phase change, both the solid and the liquid phases are present and are separated by a moving interface between them. The difficulty in solving a phase change problem is the presence of a moving boundary or region in which heat and mass balance conditions have to be met.

\section{Methodology}

\subsection{System Descriptions}

The scheme of the architectural configuration of the system is shown in Fig. 1a. It consists of 2 shutter panels, each $0.5 \mathrm{~m}$ by $1.5 \mathrm{~m}$ and composed of a set of several aluminium rectangular cavities filled with the PCM. The horizontal walls of the cavities are supposed to act as a fin arrangement and enhance the rate of heat transfer to the PCM. To compensate the low thermal conductivity of PCM, the use of metal fins in a latent heat storage system has been studied by many authors [7-11]. The insulation layer on the back surface of the shutter is essential to enable the control of the direction of the stored heat delivery, especially during the thermal discharge of the system.

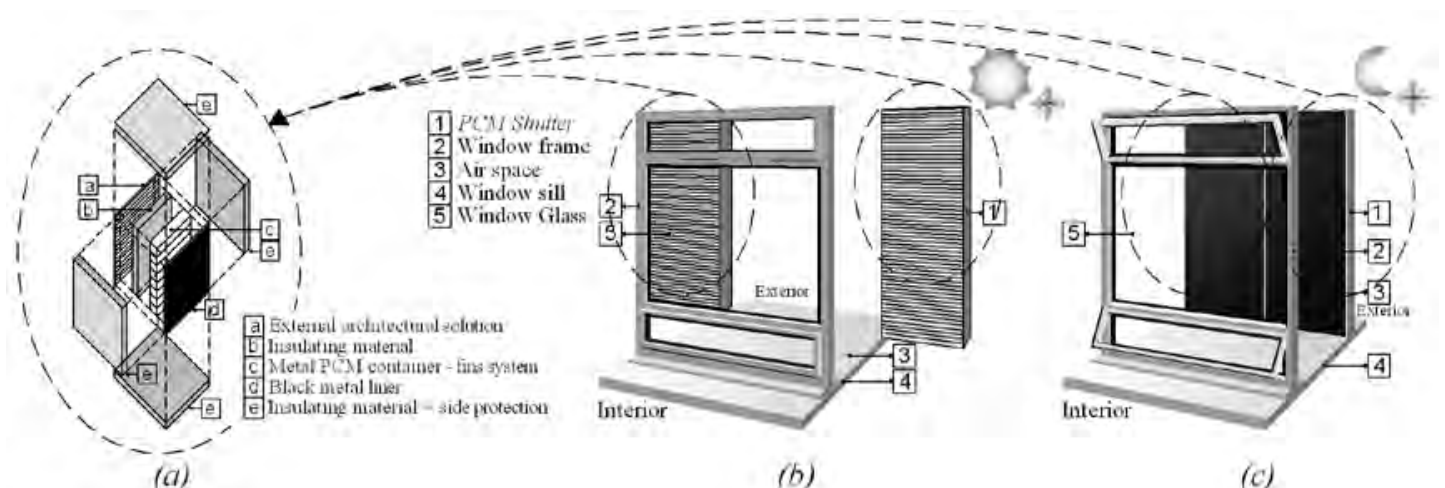

Fig. 1. (a) Schematics of the latent heat storage system; (b) Schematics of the functioning of the PCM shutter during a winter day - PCM charging when the system is opened; (b) Schematics of the functioning of the PCM shutter during a winter night - PCM discharging when the system is closed.

The rationale of the system operation during a consecutive day and night in winter is sketched in Figs. 1b and 1c. The system must operate cyclically, reflecting the ongoing cycles of the daytime and of the PCM phase change process (from solid to liquid and vice versa) and, at the same time, enabling the storage and release of thermal energy. The system is to be opened during the day to maximize the solar direct gains through the glass window and the charging of the system - PCM melting. During the night the system must be closed to minimize the heat losses through the glazing and to allow the discharging of the system by releasing the thermal energy indoors through the window arrangement - PCM solidification. During the night the window must be somehow opened to ensure the ventilation of the air cavity separating the window glass and the PCM shutter promoting the heat release indoors. 
Many authors have considered the design and analysis of a system with the same goals as the one we propose here, particularly Costa et al. [7] and Brousseau and Lacroix [8]. Both works define and study latent heat thermal energy storage systems designed to store the off-peak electrical energy in the form of thermal energy via PCM phase change processes. Using offpeak electricity, a PCM can be melted to store electrical energy in the form of latent heat thermal energy, and the heat is then available when needed for space heating, during the period when the electricity is most expensive. The system proposed in this work aims to take advantage of the solar energy, instead of the electrical energy, to melt the PCM.

\subsection{General Assumptions}

The chosen PCM for this simulation is similar to an n-Octadecane - CH3(CH2)16CH3. The thermophysical properties of this PCM and of the heat exchanger material (aluminium) are given in Table 1. One uses all of n-Octadecane's thermophysical properties, except for the melting temperature, which shall be considered lower than that of n-Octadecane and closer to indoor comfort temperature in wintertime.

Table1. Thermophysical properties of the system materials.

\begin{tabular}{lcc}
\hline \multicolumn{1}{c}{ Thermophysical properties } & PCM & Aluminium \\
\hline Latent heat of fusion $\left(\mathrm{J} \mathrm{kg}^{-1}\right)$ & 243500 & - \\
Density - solid $\left(\mathrm{kg} \mathrm{m}^{-3}\right)$ & 865 & 2707 \\
Density - liquid $\left(\mathrm{kg} \mathrm{m}^{-3}\right)$ & 780 & - \\
Specific heat - solid $\left(\mathrm{J} \mathrm{kg}^{-1}{ }^{\circ} \mathrm{C}^{-1}\right)$ & 1934 & 896 \\
Specific heat - liquid $\left(\mathrm{J} \mathrm{kg}^{-1} \mathrm{C}^{-1}\right)$ & 2196 & - \\
Thermal conductivity - solid $\left(\mathrm{W} \mathrm{m}^{-1}{ }^{\circ} \mathrm{C}^{-1}\right)$ & 0.358 & 204 \\
Thermal conductivity - liquid $\left(\mathrm{W} \mathrm{m}^{-1}{ }^{\circ} \mathrm{C}^{-1}\right)$ & 0.148 & - \\
\hline
\end{tabular}

The main reference outdoor conditions, Eq. (1), are the external air temperature, $T_{e, r e f}$, and the solar radiation in a vertical South facing wall in Coimbra in January, $\dot{q}_{\text {rad,ref }}$, both calculated according to the climatic data of SOLTERM ${ }^{\circledR}$ during the period when the system must be opened - from 9 am until $5 \mathrm{pm}$. The external convection heat transfer coefficient considered is $h_{e}=25 \mathrm{~W} \mathrm{~m}^{-2}{ }^{\circ} \mathrm{C}^{-1}$.

$\left\{\begin{array}{l}T_{e, \text { ref }}=8.2-5.5 \sin (0.32 t) \\ \dot{q}_{\text {rad, ref }}=160.0-350.0 \sin (0.385 t)\end{array}\right.$, with $t$ in hours and $\theta$ in rad

The indoor air temperature, $T_{i}$, is influenced by outdoor conditions, by the internal loads and by the buildings envelope constitution. However in this study, during the time when the system must be closed - from $5 \mathrm{pm}$ until 9 am - the average indoor air temperature is considered fixed and equal to $15^{\circ} \mathrm{C}$. The internal convection heat transfer coefficient, $h_{i}$, can vary according to the model chosen for its determination, as explained below.

\subsection{Numerical Simulation and Numerical Solution}

The model was developed using the enthalpy formulation, as sketched in Fig. 2. In the discretized energy conservation equation, the volumetric sensible enthalpy, $h$, is the dependent variable and the latent energy rate involved in the phase change process is considered in the source term, which in turn is expressed in terms of the local fraction of melted PCM. The main advantages of this method are: (i) the dispense of a condition to be 
satisfied at the solid-liquid interface (as it automatically obeys the interface condition), (ii) the existence of a mushy zone between the two phases in each control volume, (iii) the fact that the energy conservation equation is similar to the one of the pure heat diffusion model (without phase change) and (iv) that this method guaranties the isothermal nature of the phase change process. This model is meant to solve the problem of heat diffusion associated to the PCM phase change in a 2-dimensional domain. The differential equation governing the underlying physical phenomenon is integrated using the finite-volume method proposed by Patankar [12]. The system of equations is iteratively solved by a tri-diagonal-matrix algorithm and the evolution in time is modelled according to a totally implicit formulation. Further details concerning the numerical implementation of present enthalpy method may be found in the previous work of the authors [13].

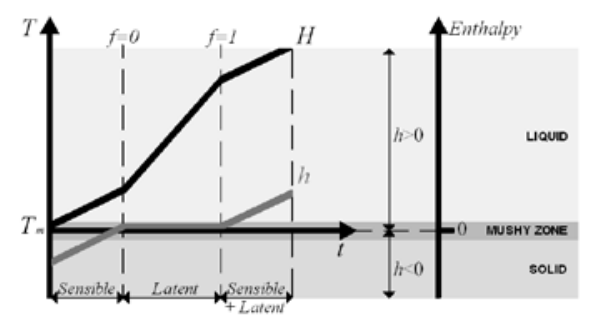

Fig. 2. Sketch of the enthalpy formulation.

\section{Results and Discussion}

This section is devoted to the identification of the optimal PCM volume, and of the optimal PCM melting temperature, $T_{m}$. The first part shows the existence of the optimum PCM volume and its calculation for a specific case. The following parts are concerned with the estimation of the impact of some parameters: outdoor condition, indoor conditions and PCM thermophysical properties. Finally, the optimal configuration is discussed.

\subsection{Determination of the PCM volume}

Case of: $\left\{\begin{array}{l}T_{m}=18{ }^{\circ} \mathrm{C}, L=243500 \mathrm{~J} \mathrm{~kg}^{-1}, h_{i} \text { estimated by natural convection over vertical surface } \\ T_{i}=15{ }^{\circ} \mathrm{C}, h_{e}=25 \mathrm{~W} \mathrm{~m}^{-2}{ }^{\circ} \mathrm{C}^{-1}, T_{e}=T_{e, r e}, \dot{q}_{\text {rad }}=\dot{q}_{\text {rad }, \text { ref }}, C M=R v i=0.002 \mathrm{~m}, \mathrm{FL}=0.03 \mathrm{~m}\end{array}\right.$

In order to find the optimal PCM volume to incorporate in the shutter, a set of numerical experiments was held using the methodology presented in Section 2.3, for the test case described in Section 2.1 and for the values above. The optimum PCM volume corresponds to the one that can be totally melted during the charging period (daytime) and totally solidified during the discharging period. Due to the low thermal diffusivity of the PCM, the total volume melted and solidified is strictly related with the distance between the metal fins.

For a given inter-fins distance $H L$, the enthalpy of the system, $H$, and the total PCM melted fraction, $F$, were computed. Figure 3a shows that the PCM can be totally melted considering $H L=0.004 \mathrm{~m}$. This means that the total volume of PCM with which the container described in Section 2.1 can be filled represents $40 \%$ of the total volume of the system. Results with higher PCM volumes (larger distances between fins) are not so attractive because they cannot be totally melted during the day, and so, the enthalpy of the system is smaller (Fig. 3b) and the PCM potential as a latent heat material is overvalued. The results considered in Fig. 3a and in Fig. 3b were obtained for three consecutive day cycles numerically simulated, starting from a condition of no PCM melted and the whole system at a uniform temperature $T_{m}$. 
For the PCM volume corresponding to $H L=0.004 \mathrm{~m}$ the discharging process is not completed during the night, which can mean that there will be an enduring melted PCM fraction overvaluing the potential of PCM as a latent heat material. In order to enhance the discharging of the system and the solidifying process of the PCM, indoor forced airflow must be induced in the air gap between the shutter and the glass to enhance heat convection in that period.

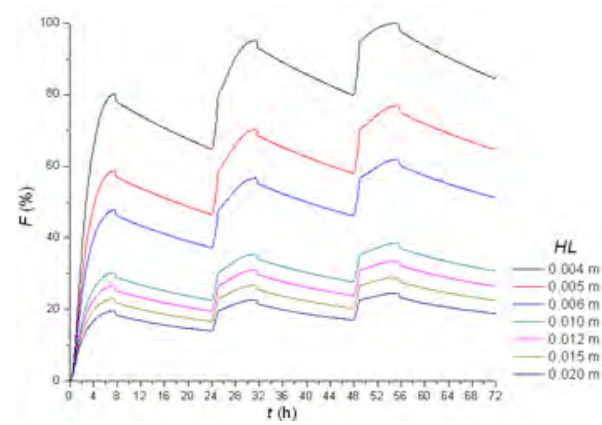

(a)

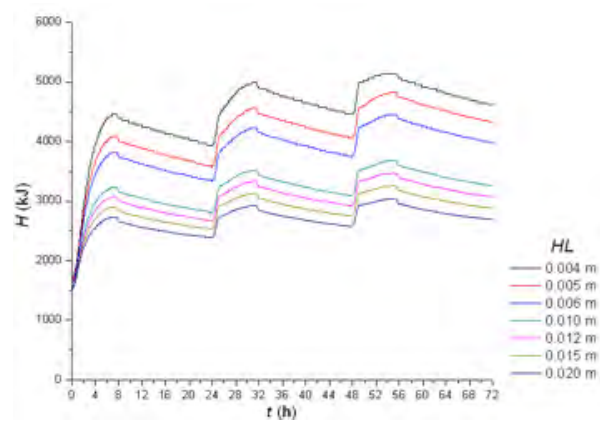

(b)

Fig. 3. Evolutions (a) of the total PCM melted fraction, F, and (b) of the enthalpy of the system, $H$, during three consecutive day cycles simulated.

\subsection{Impact of the internal heat transfer coefficient}

Case of: $\left\{\begin{array}{l}T_{m}=18{ }^{\circ} \mathrm{C}, L=243500 \mathrm{~J} \mathrm{~kg}^{-1}, T_{i}=15^{\circ} \mathrm{C}, h_{e}=25 \mathrm{~W} \mathrm{~m}^{-2}{ }^{\circ} \mathrm{C}^{-1} \\ T_{e}=T_{e, \text { ref }}, \dot{q}_{\text {rad }}=\dot{q}_{\text {rad }, \text { ref }}, C M=R v i=0.002 \mathrm{~m}, \mathrm{FL}=0.03 \mathrm{~m}, \mathrm{HL}=0.004 \mathrm{~m}\end{array}\right.$

A set of numerical experiments was held in order to evaluate the influence of the $h_{i}$ value on the total energy stored in the system via the PCM phase change from solid to liquid and released by the system to the indoor air via PCM phase change from liquid to solid. The different values for $h_{i}$ considered in the simulations are shown in the legend of Fig. 4.

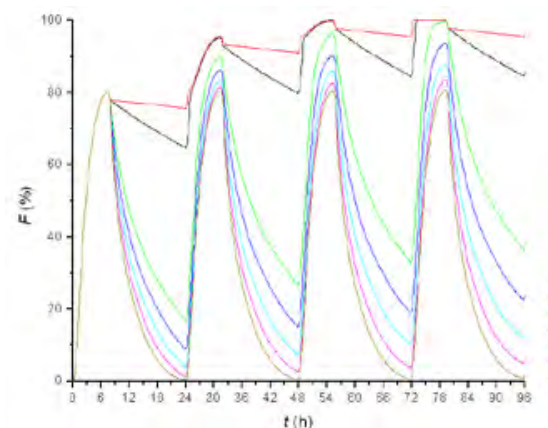

(a)

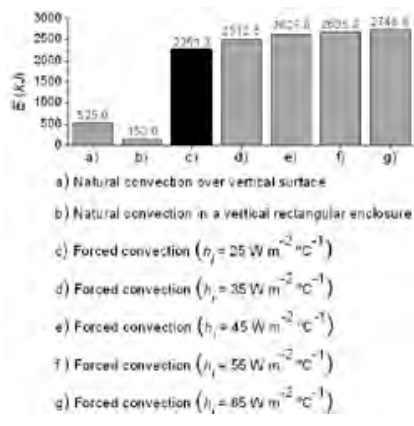

(b)

Fig. 4. (a) Evolution of the F value, during the first four numerical cycles for different values of $h_{i}$; (b) Evaluation of the $E$ value during the fourth simulated cycle for the different models considered for $h_{i}$.

The results presented in Fig. 4a were obtained for the first four numerical day cycles simulated, the point in time when steady cyclic behaviour was achieved. The values of the total energy stored and released indoors by the system during a complete day cycle, $E$, showed in Fig. 4b, correspond to those of the fourth day cycle simulated. By matching the results of Fig. $4 \mathrm{a}$ and Fig. $4 \mathrm{~b}$ one can see that the $E$ value is proportional to the fraction of PCM solidified during the discharging period. The larger the value of $h_{i}$ the larger is the fraction of PCM solidified during the night and the heat released to the indoor space. For very large values of $h_{i}$ the system is totally discharged but the PCM volume cannot be totally 
melted during the day. Fixing $h_{i}=h_{e}=25 \mathrm{~W} \mathrm{~m}^{-2}{ }^{\circ} \mathrm{C}^{-1}$ one can enhance the performance of the system during the night without compromising the melted fraction during the day.

\subsection{Impact of the indoor temperature}

Case of: $\left\{\begin{array}{l}T_{e}=T_{e, r e}, \dot{q}_{\text {rad }}=\dot{q}_{\text {rad }, \text { ref }}, h_{i} \text { estimated by natural convection over vertical surface } \\ h_{e}=25 \mathrm{~W} \mathrm{~m}^{-2}{ }^{\circ} \mathrm{C}^{-1}, L=243500 \mathrm{~J} \mathrm{~kg}^{-1}, C M=R v i=0.002 \mathrm{~m}, F L=0.03 \mathrm{~m}, \mathrm{HL}=0.004 \mathrm{~m}\end{array}\right.$

In order to evaluate de impact of the indoor temperature considered, $T_{i}$, a set of numerical experiments was held with a range of values for $T_{i}$ and $T_{m}$. The results are shown in Fig. 5 . One can see that the $E$ value grows proportionally to the difference between $T_{m}$ and $T_{i}$. That is because the driving potential for the heat flow rate to the inside is enhanced and the release of energy during the night is improved while the PCM changes phase from liquid to solid.

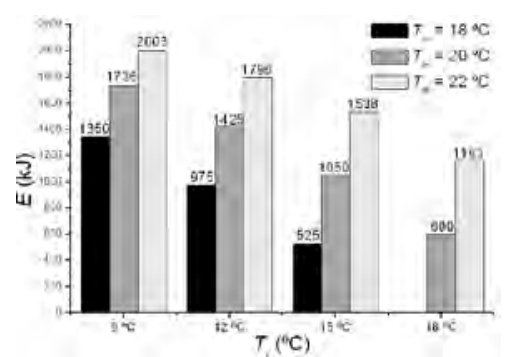

Fig. 5. Evaluation of the total energy stored and released by the system, E, during the fourth numerical day cycle for the different values considered for $T_{m}$ and $T_{i}$.

\subsection{Impact of the outdoor conditions}

Case of: $\left\{\begin{array}{l}L=243500 \mathrm{~J} \mathrm{~kg}^{-1}, T_{m}=18{ }^{\circ} \mathrm{C}, h_{i} \text { estimated by natural convection over vertical surface } \\ h_{e}=25 \mathrm{~W} \mathrm{~m}^{-2}{ }^{\circ} \mathrm{C}^{-1}, T_{i}=15^{\circ} \mathrm{C}, \mathrm{CM}=\mathrm{Rvi}=0.002 \mathrm{~m}, \mathrm{FL}=0.03 \mathrm{~m}, \mathrm{HL}=0.004 \mathrm{~m}\end{array}\right.$

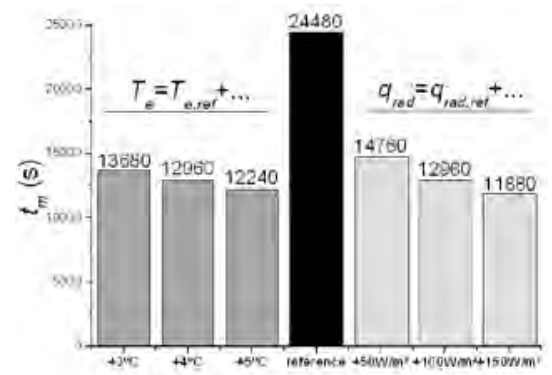

Fig. 6. Evaluation of the time needed to melt all the PCM volume, $t_{m}$, considering the reference outdoor conditions and 3 different sinusoidal swing approaches to calculate $T_{e}$ and $\dot{q}_{\text {rad }}$ evolutions.

The impact of the outdoor air temperature, $T_{e}$, is evaluated through the time needed to melt all the PCM volume, $t_{m}$, using three different sinusoidal swing approaches to estimate the $T_{e}$ evolution during the time when the system must be opened, and comparing them with the reference sinusoidal curve presented in Section 2.2. The same methodology is carried out for the solar radiation, $\dot{q}_{\text {rad }}$. The results are presented in Fig.6 showing that the higher the outdoor air temperature and the solar radiation are, the less time it takes to melt all the PCM volume.

\subsection{Impact of the PCM melt temperature}

Case of: $\left\{\begin{array}{l}L=243500 \mathrm{~J} \mathrm{~kg}^{-1}, T_{i}=15^{\circ} \mathrm{C}, h_{i}=h_{e}=25 \mathrm{~W} \mathrm{~m}^{-2}{ }^{\circ} \mathrm{C}^{-1} \\ T_{e}=T_{e, r e f}, \dot{q}_{\text {rad }}=\dot{q}_{\text {rad }, \text { ref }}, C M=R v i=0.002 \mathrm{~m}, \mathrm{FL}=0.03 \mathrm{~m}, \mathrm{HL}=0.004 \mathrm{~m}\end{array}\right.$ 
The total energy stored and released by the system, $E$, is strongly influenced by the melt temperature of the PCM, $T_{m}$. When it is lower than the optimum $T_{m}$, the charging process is faster and less time is needed to complete it, thereby melting all the PCM mass. On the other hand, if the difference between $T_{m}$ and $T_{i}$ is very small, the discharging process of the system is compromised and the time needed to complete it is larger than the time available (period of system closed). When $T_{m}$ is higher than the optimum temperature, the discharging process is more efficient. However, the charging (melting) process will not be completed in the time specified by the operating conditions. Figure 7 illustrates the influence of $T_{m}$ in the $E$ value.

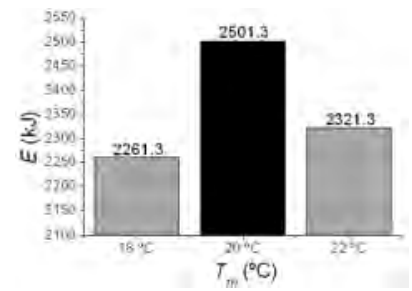

Fig. 7. Influence of $T_{m}$ in the total energy stored by the system during the day-time and released to the interior during the night in a complete day cycle under the same operating conditions.

\subsection{Existence of the optimum}

Case of: $\left\{\begin{array}{l}T_{m}=20{ }^{\circ} \mathrm{C}, L=243500 \mathrm{~J} \mathrm{~kg}^{-1}, T_{i}=15^{\circ} \mathrm{C}, h_{i}=h_{e}=25 \mathrm{~W} \mathrm{~m}^{-2}{ }^{\circ} \mathrm{C}^{-1}, \\ T_{e}=T_{e, r e f}, \dot{q}_{\text {rad }}=\dot{q}_{\text {rad }, \text { ref }}, C M=R v i=0.002 \mathrm{~m}, \mathrm{FL}=0.03 \mathrm{~m}, \mathrm{HL}=0.004 \mathrm{~m}\end{array}\right.$

The reference test case above shows an optimum configuration for the system. The total energy stored and released by the system, $E$, during a complete 24 hours day cycle is compared with that of other $0.03 \mathrm{~m}$ thick building materials under the same operating conditions. The results are shown in Fig. 8.

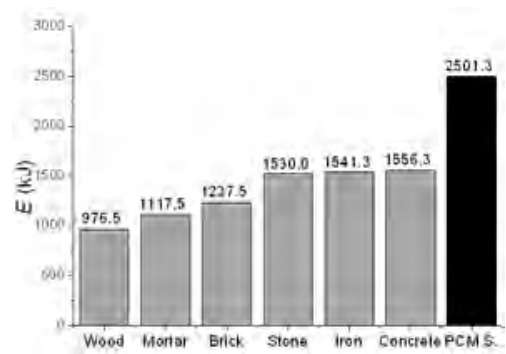

Fig. 8. Total energy stored and released by the system during a complete day cycle, E, compared it with that of other $0.03 \mathrm{~m}$ thick building materials under the same operating conditions.

\section{Conclusions}

The outdoor air temperature and the solar radiation flux play an important role during melting and consequently have a strong effect on the time needed to melt the PCM volume. On the other hand, the indoor temperature and the convection heat transfer rate on the inner side of the shading element, during the night, play an important role for the PCM solidification and have a strong effect on the energy release indoors. To enhance the discharging of the PCM a ventilator must be adjoined to the inner side to increase the internal heat transfer coefficient. In conclusion, using the numerical simulation as carried out, an optimal system configuration, with an optimal PCM melting temperature, can be found for any given location and characteristic climatic data during the winter season. The results for stored and released energy in a complete day cycle, using a $0.03 \mathrm{~m}$ thick layer of different materials, show the 
enhancement of the thermal inertia of the building envelope obtained by using the system described. The Tm value has a strong influence on the energy storage capacity of the system. The optimal melting temperature of the PCM to be incorporated in the system for the location of Coimbra is $20^{\circ} \mathrm{C}$. According to the present predictions, the total energy stored and released by the optimum system during a complete 24 hours day cycle can reach $2501.3 \mathrm{~kJ}$.

Further work has to be done to assess the behaviour of the system in a physical testing prototype and cell test. A building dynamic simulation has also to be carried out to evaluate the influence of the latent heat loads in the thermal behaviour of the building.

\section{References}

[1] V.V. Tyagi \& D. Buddhi, 'PCM thermal storage in buildings: A state of art', Renewable and Sustainable Energy Reviews, 11, 2007, pp. 1146-1166.

[2] V.V. Tyagi, S.C. Kaushik, S.K. Tyagi \& T. Akiyama, 'Development of phase change materials based microencapsulated technology for buildings: A review', Renewable and Sustainable Energy Reviews, 2010.

[3] B. Zalba, J.M. Marín, L.F. Cabeza \& H. Mehling, 'Review on thermal energy storage with phase change: materials, heat transfer analysis and applications', Applied Thermal Engineering, 23, 2003, pp. 251-283.

[4] A. Sharma, V.V. Tyagi, C.R. Chen \& D. Buddhi, 'Review on thermal energy storage with phase change materials and applications', Renewable and Sustainable Energy Reviews, 13, 2009, pp. 318-345.

[5] A. Pasupathy, R. Velraj \& R.V. Seeniraj, 'Phase change material-based building architecture for thermal management in residential and commercial establishments', Renewable and Sustainable Energy Reviews , 12, 2008, pp. 39-64.

[6] C.R. Swaminathan \& V.R. Voller, 'On the enthalpy method', International Journal of Numerical Methods for Heat \& Fluid Flow, 3, 1993, pp. 233-244.

[7] M. Costa, D. Buddhi \& A. Oliva, 'Numerical simulation of a latent heat thermal energy storage system with enhanced heat conduction', Energy Conversion and Management, 39[3/4], 1998, pp. 319-330.

[8] P. Brousseau \& M. Lacroix, 'Numerical simulation of a multi-layer latent heat thermal energy storage system’, International Journal of Energy Research, 22, 1998, pp. 1-15.

[9] C.R. Chen \& A. Sharma, 'Numerical investigation of melt fraction of PCMs in a latent heat storage system', Journal of Engineering \& Applied Sciences, 1, 2006, pp. 437-444.

[10]A. Sharma, S.D. Sharma, D. Buddhi \& L.D. Won, 'Effect of thermo physical properties of heat exchanger material on the performance of latent heat storage system using an enthalpy method', International Journal of Energy Research, 30, 2006, pp. 191-201.

[11]C.R. Chen, A. Sharma, S.K. Tyagi \& D. Buddhi, 'Numerical heat transfer studies of PCMs used in a box-type solar cooker’, Renewable Energy, 33, 2008, pp.1121-1129.

[12]S.V. Patankar, Numerical heat transfer and fluid flow, Hemisphere Publishing Corporation, Washington, 1980.

[13] N. Soares, R. Vicente, A. Samagaio \& J. Costa, 'Investigation of a multi-layer latent heat solar thermal energy storage system with PCMs', Proceeding of $12^{\text {th }}$ International Conference on Building Materials and Components, 2011. 